International Journal of Canadian Studies

Revue internationale d'études canadiennes

INTERNATIONAL JOURNAL OF CANADIAN STUDIES

REYUE INTERNATIONALE D'ÉTUDES CANADIENNES

\title{
L'eau du Canada : une ressource stratégique dans le cadre du PSP
}

\section{Delia Montero}

Numéro 39-40, 2009

Culture - Natures in Canada

Culture - natures au Canada

URI : https://id.erudit.org/iderudit/040833ar

DOI : https://doi.org/10.7202/040833ar

Aller au sommaire du numéro

\section{Éditeur(s)}

Conseil international d'études canadiennes

\section{ISSN}

1180-3991 (imprimé)

1923-5291 (numérique)

Découvrir la revue

Citer cet article

Montero, D. (2009). L'eau du Canada : une ressource stratégique dans le cadre du PSP. International Journal of Canadian Studies / Revue internationale d'études canadiennes, (39-40), 283-300. https://doi.org/10.7202/040833ar
Résumé de l'article

La question de l'eau a pris de l'importance au cours des dernièresannées, non seulement en raison de sa disponibilité et de l'augmentation dela demande due à l'accroissement des grands conglomérats urbains et dudéveloppement industriel, mais aussi parce que les accords de libre-échange ontouvert la voie à sa commercialisation. Les débats portent sur la gestion publique ouprivée de la ressource, sa privatisation et sa commercialisation. Somme toute,l'eau du Canada est devenue un sujet de première importance à cause, d'une part, de sa grande abondance et, d'autre part, de la demandecroissante des États-Unis, son principal partenaire. Le but de cet article estd'analyser l'évolution dans les relations entre les deux pays enmatière d'eau ainsi que les nouvelles négociations dans le cadre du PSP.
Tous droits réservés @ Conseil international d'études canadiennes, 2009
Ce document est protégé par la loi sur le droit d'auteur. L'utilisation des services d'Érudit (y compris la reproduction) est assujettie à sa politique d'utilisation que vous pouvez consulter en ligne.

https://apropos.erudit.org/fr/usagers/politique-dutilisation/ 


\title{
Delia Montero
}

\section{L'eau du Canada : Une ressource stratégique dans le cadre du PSP}

\begin{abstract}
Résumé
La question de l'eau a pris de l'importance au cours des dernières années, non seulement en raison de sa disponibilité et de l'augmentation de la demande due à l'accroissement des grands conglomérats urbains et du développement industriel, mais aussi parce que les accords de libre-échange ont ouvert la voie à sa commercialisation. Les débats portent sur la gestion publique ou privée de la ressource, sa privatisation et sa commercialisation. Somme toute, l'eau du Canada est devenue un sujet de première importance à cause, d'une part, de sa grande abondance et, d'autre part, de la demande croissante des Etats-Unis, son principal partenaire. Le but de cet article est d'analyser l'évolution dans les relations entre les deux pays en matière d'eau ainsi que les nouvelles négociations dans le cadre du PSP.
\end{abstract}

\begin{abstract}
Water has become more important over the past years, not only because of increasing consumption by large urban conglomerates, industrial development, and its availability, but also because free-trade agreements open up commercial possibilities. This has fuelled debate regarding public and private management, privatization and commercialization. In this perspective, Canada's water is a subject of prime importance, not only because of its enormous availability, but also because of growing demand from the United States, its principal partner. This article aims to analyze how the "water" relationship between Canada and the United States developed, as well as the new negotiations within the SPP.
\end{abstract}

Le problème de l'eau au Canada a pris de l'importance au cours des trois dernières décennies en raison de l'accroissement de la consommation par les grands centres urbains, de l'expansion et de la vitalité du secteur industriel et de la valeur commerciale de l'eau. Le débat porte sur deux questions. Est-ce que l'eau est un bien public dont la gestion devrait rester entre les mains de l'État? Quelles règles régissent le commerce de l'eau?

Or, la gestion de l'eau du Canada, une ressource stratégique importante, représente une des priorités dans l'élaboration de l'agenda du Partenariat nord-américain pour la sécurité et la prospérité (PSP) signé en mars 2005 par les chefs d'État du Mexique, des États-Unis et du Canada. Évidemment, les États-Unis, qui consomment d'importantes quantités de cette ressource, sont le pays signataire le plus intéressé à assurer son approvisionnement en eau. La question de l'eau a toujours été importante dans les relations entre le 
Canada et les États-Unis parce que les réserves d'eau partagées par ces deux pays, notamment les Grands Lacs, figurent parmi les plus importantes du monde. Depuis les années soixante, la question revêt une importance encore plus grande puisqu'on parle maintenant d'exporter de l'eau en vrac vers les États-Unis.

Avec la signature du PSP, la question a resurgi, cette fois comme composante d'une stratégie continentale, prétextant la prospérité et la sécurité des pays signataires. Il va sans dire que dans cet accord, l'eau acquiert une toute nouvelle signification dans les relations entre le Canada et les États Unis. Effectivement, les États-Unis sont de grands consommateurs d'eau et bien qu'ils aient mis sur pied des programmes pour diminuer leur consommation, celle-ci figure toujours parmi les plus élevées au monde. Ils voient le Canada comme une solution possible à leurs problèmes d'approvisionnement et, pour cette raison, le gouvernement américain fera de l'eau une priorité dans les discussions du PSP. La question qui se pose est de savoir si le gouvernement canadien pourra résister aux pressions exercées par les Américains.

Rappelons que la question a été soulevée dans les accords de libre-échange que le Canada a signés d'abord avec les États-Unis en 1988 et plus tard avec ce même pays et le Mexique en 1994 (Accord de libre-échange de l'Amérique du Nord, ALENA), et bien que l'on ait recommandé de ne pas inclure l'eau, le gouvernement canadien n'a pas formellement demandé qu'on l'exclut des négociations comme l'a fait le Mexique avec le pétrole.

Nous soutenons que l'absence d'une volonté politique, de la part du gouvernement fédéral canadien, d'interdire explicitement les exportations d'eau vers les États-Unis au moment de la signature des accords commerciaux, s'explique par l'important rapport économique structurel entre les deux pays, rapport sur lequel repose une bonne partie de l'économie canadienne. Même si le PSP vise l'intégration autre que commerciale en Amérique du Nord, il constitue une grave menace pour la préservation de la souveraineté canadienne sur l'eau puisque, dans les faits, il s'agit d'un processus non formalisé pour aboutir à un nouveau traité international sans l'approbation du pouvoir législatif, duquel relève le contrôle des ressources naturelles dont fait partie l'eau.

Bien qu'il existe au Canada une politique nationale de l'eau qui date de 1987, celle-ci ne régule pas, à strictement parler, l'exportation de cette ressource. De plus, la Constitution du Canada prévoit une division des pouvoirs fédéraux et provinciaux et la responsabilité directe de la gestion des ressources naturelles revient aux gouvernements provinciaux, créant un vide quant à une politique commune et obligatoire pour la gestion commerciale de l'eau. Il y a donc un risque que l'eau canadienne devienne 
une marchandise exportable dans le cadre du PSP. Dans la première partie de cette note de recherche, on analysera l'évolution de la relation Canada-États-Unis sur la question de l'eau, en passant par les accords et les désaccords, particulièrement par rapport au lien structurel du Canada avec son partenaire commercial le plus important. La deuxième partie portera plus précisément sur le PSP et sur ce qui a été convenu en matière d'eau. Dans la troisième partie, on tentera de faire un bilan de la position du Canada sur cette question.

\section{La relation bilatérale entre le Canada et les États-Unis en matière hydrique}

Bien qu'au cours de la dernière décennie, la sécurité soit devenue une préoccupation majeure dans les relations canado-états-unisiennes, le maintien de bonnes relations bilatérales sur les plans politique et économique avec son voisin du sud a toujours été une des grandes priorités du gouvernement canadien. Étant donnél'importance des enjeux politiques et économiques de ces relations, la stabilité est un facteur incontournable. En plus de partager une des plus grandes frontières du monde, le long de laquelle habite $80 \%$ de la population canadienne, le Canada et les États-Unis partagent, dans les Grands Lacs, les plus grandes réserves d'eau douce du monde ( $22 \%$ de l'eau douce de surface de la planète).

La proximité des deux pays, une langue commune, les investissements transfrontaliers et les ressources naturelles figurent parmi les facteurs qui ont contribué à un commerce bilatéral très dynamiquel et, évidemment, à une importante coopération entre le Canada et les États-Unis. On retrouve plusieurs exemples de cette coopération dans les accords, tels la Déclaration sur la frontière intelligente de $2001^{2}$, l'établissement du Groupe de planification binationale en 2002 et le Partenariat nord-américain pour la sécurité et la prospérité de 2005 . Or, la défense, le commerce et le développement ont traditionnellement été les trois principales préoccupations des discussions binationales, depuis même le premier traité de réciprocité du $19^{\mathrm{e}}$ siècle. Ces trois sujets, qui sont d'un grand intérêt pour les États-Unis, figurent en effet dans les traités récents, mais revêtent un aspect plus global encore dans le PSP de 2005. Ainsi la relation bilatérale entre le Canada et les États-Unis est plus qu'une relation économique; elle est devenue aussi une relation stratégique. Le Canada et les États-Unis cherchent, ạvec le Mexique, à présenter un front commun, particulièrement en ce qui concerne la sécurité territoriale, ce qui comprend bien entendu la suffisance énergétique et des ressources naturelles telle que l'eau.

La relation bilatérale entre le Canada et les États-Unis a été renforcée par l'actuel premier ministre conservateur Stephen $\mathrm{Harper}^{3}$, dont le discours et les actions tendent à une plus grande intégration des deux pays. En effet, $\mathbf{M}$. Harper a laissé une forte impression, à plusieurs reprises, par ses 
déclarations et ses actions, d'un alignement total avec le courant néoconservateur qui domine aux États-Unis. Effectivement, dès son arrivée au pouvoir, il a laissé entrevoir que la grande priorité de la politique extérieure de son gouvernement conservateur serait les rapports avec les États-Unis, une préoccupation omniprésente que certains qualifient de relation d'interdépendance complexe. La politique de $M$. Harper penche évidemment vers une plus grande intégration économique avec les États-Unis, ce qu'on a qualifié de «deep integration», intégration qui déborde le secteur économique pour se faire ressentir dans les décisions dans les domaines politique, socioculturel, environnemental et de la sécurité 4 .

En matière économique et commerciale, on considère, entre autres, une union monétaire, un accord de gestion partagée des ressources naturelles le Canada étant le principal exportateur d'énergie aux États-Unis (gaz et pétrole) - un marché continental de l'eau et une plus grande intégration verticale au détriment des relations interprovinciales.

Il est clair qu'une des priorités du gouvernement américain est de s'assurer du contrôle des ressources naturelles, ${ }^{5}$ principalement de l'énergie et de l'eau dont dispose le Canada. Ces deux ressources représentent un élément clé pour les pays du nord (Serfati, 2006). Une priorité connexe est d'assurer que les investissseurs étrangers ont accès aux secteurs liés aux ressources naturelles, dontl'eau et le pétrole. Cette liberté d'accès est importante pour les grandes entreprises américaines.

Dans le cas particulier de la gestion des eaux limitrophes, dont l'eau des Grands Lacs, le Canada et les Etats-Unis ont une longue histoire marquée par des accords et des désaccords ${ }^{6}$. Entamées au siècle dernier, les discussions à ce sujet ont mené à la signature, en 1909, du Traité relatif aux eaux limitrophes. Cette entente administrée par la Commission mixte internationale (CMI), répartit les responsabilités de base et établit les objectifs par rapportà la gestion des cours d'eau et des lacs qui constituent la frontière naturelle entre les territoires. (OCDE, 2004 179). En 1972, le Canada et les États-Unis ont signé l'Accord relatifà la qualité de l'eau des Grands Lacs, lequel a été révisé en 1978 et en 1987. Cet accord représente un départ par rapport à l'engagement de maintenir l'intégrité de l'écosystème des Grands Lacs (Montero, 2006) ${ }^{7}$. Il a donné lieu à deux nouveaux accords en 2005. Dans ces accords bilatéraux, on n'ạ pas abordé la question de l'exportation de l'eau de manière formelle. Toutefois, ce que l'on a discuté, ce sont les nombreuses disputes au sujet des déviations d'eau vers les États-Unis ${ }^{8}$, disputes liées à l'accroissement des quotas quant à la consommation d'eau de ce pays'. Il faut signaler que dans l'Ouest américain, le développement économique, la croissance démographique et même le mode de vie des gens reposent sur une importante consommation d'eau. Des conflits relatifs au partage de l'eau dans cette zone ont surgi 
parce que le débit de la rivière Colorado ne suffit pas à approvisionner en eau les États qu'elle traverse.

C'est ainsi qu'en 1922, les délégués de sept États de l'union américaine (le Wyoming, le Colorado, le Utah, le Nouveau Mexique, l'Arizona, le Nevada et la Californie) ont signé l'accord Colorado River Compact, fixant la quantité d'eau à laquelle avait droit chacun des États. (Lasserre 370) Les conflits portant sur la répartition de l'eau de la rivière Colorado soulignent l'importance de construire des aqueducs pour le transport de l'eau canadienne vers les États-Unis. L'accès à l'eau canadienne devient alors un sujet de premier ordre pour les États-Unis. D'ailleurs, l'Organisation de coopération et de développement économique (OCDE) considère que le Canada dispose de beaucoup d'eau. Cependant, si l'on compare la disponibilité de l'eau dans ces deux pays par rapport à l'ensemble du monde, on observe que le Canada et les Etats-Unis disposent de réserves semblables $(6,5 \%$ et $6,4 \%$ respectivement). Par contre, si ces réserves sont calculées à partir de la population de ces deux pays, le Canada dispose de beaucoup plus d'eau que les États-Unis puisque sa population n'est que d'un peu plus de 33 millions d'habitants. Si le Canada détient $22 \%$ du total mondial des réserves d'eau, il faut reconnaître qu'une grande partie de cette eau n'est pas facilement 'disponible' puisqu'elle se trouve dans des glaciers, dans des couches souterraines, d'où le fait que le Canada n'a que $6,5 \%$ des réserves disponibles du monde. Néanmoins, ces réserves sont convoitées par le gouvernement américain et les chefs d'entreprises.

Au-delà des accords impliquant la gestion de l'eau des Grands Lacs, on observe trois moments importants dans les relations politiques et économiques canado-étatsuniennes, où la position du gouvernement canadien sur la possibilité de commercialiser son eau ne semble pas avoir été suffisamment claire. Le premier moment correspond aux démarches qui ont mené à la signature de l'Accord de libre-échange entre le Canada et les États-Unis (ALÉ) en 1989, le deuxième à la signature de l'ALENA en 1994, et le troisième à la signature du PSP en 2005.

La première fois que la question de l'exportation de l'eau vers les États-Unis a été abordée remonte aux années soixante-dix, alors que l'on discutait de l'idée de construire un grand canal (Grand Canal Company) pour transporter l'eau canadienne vers le sud. Ces discussions qui se sont déroulées dans un contexte marqué par le commerce croissant entre les deux nations n'ont jamais débouché sur la signature d'un accord et le débat sur l'accès à l'eau canadienne a été reporté. ${ }^{10}$ Plus tard, en août 1988 , envisageant la possibilité d'un accord de libre-échange avec les États-Unis, le ministre de l'Environnement de l'époque, l'honorable Tom McMillan, a déposé à la Chambre des communes le projet de loi C-156 intitulé Loi sur la préservation de l'eau au Canada. Ce projet de loi était fondé sur la responsabilité constitutionnelle du gouvernement fédéral. Quoique la 
Constitution canadienne confère aux provinces la responsabilité de gérer les ressources naturelles, dont l'eau, lorsqu'il est question d'exportation, le gouvernement fédéral doit exercer son «pouvoir résiduel» pour préserver l'intérêt national. C'est à partir de cette responsabilité constitutionnelle que le ministre McMillan avait énoncé la position du gouvernement canadien en matière d'exportation de l'eau douce. Selon cette politique, le gouvernement du Canada s'opposait catégoriquement aux exportations d'eau en grandes quantités.

Par ailleurs, le projet de loi C-156 prévoyait la signature d'ententes entre les gouvernements nationaux et provinciaux pour l'octroi de licences permettant l'exportation de petites quantités d'eau par navire-citerne ou par canalisation. L'octroi d'une licence d'exportation aurait exigé une étude environnementale approfondie. Les exportations minimes telles l'eau utilisée dans les produits manufacturés ou l'eau en bouteille auraient échappé à cette loi. Le projet de loi est mort au feuilleton avec la dissolution du Parlement le $1^{\text {er }}$ octobre 2008. Fait à noter, aucun projet semblable, aucune initiative ministérielle, n'a été déposé depuis à la Chambre des communes (Johansen, 1999).

Le deuxième moment important correspond à la signature de l'ALENA. Puisque le Canada n'a pas formellement demandé que l'eau soit exclue de l'accord, la possibilité existe toujours que l'eau soit considérée comme une marchandise et, par conséquent, que les signataires ne puissent en interdire ou en restreindre l'exportation ou la vente d'un pays à un autre. Si le Canada permettait l'exportation de l'eau vers les États-Unis, il lui serait impossible à l'avenir de l'interdire. Étant donné que l'ALENA accorde un traitement égal aux entreprises des trois pays, une entreprise américaine aurait les mêmes droits d'exploiter et d'exporter l'eau qu'une entreprise canadienne, ce qui pourrait mener à une situation semblable à celle du gaz et du pétrole, où les investissements américains ont augmenté de plus de $50 \%$ et de $70 \%$ respectivement, et où la plupart des exportations sont destinées au marché américain. C'est la situation actuelle avec l'eau embouteillée, où les entreprises étrangères contrôlent presque $50 \%$ de la production. (Montero, 2004).

Jean Chrétien, du Parti libéral, élu premier ministre en 1993, a été un personnage clé dans les négociations de l'ALENA. Tout comme les gouvernements conservateurs, le gouvernement libéral a refusé de négocier l'exemption de l'eau dans le texte des accords commerciaux, même si le gouvernement canadien avait négocié des exemptions pour quelques matières premières telles que le bois brut et le poisson non apprêté. Alors que le Mexique a refusé que le pétrole fasse partie des négociations de l'accord, le Canada n'a exclu l'eau que techniquement. Le cabinet du Premier ministre a émis un communiqué de presse où il indiquait que les 
trois gouvernements étaient d'accord que rien dans l'ALENA n'obligeait un des signataires à exporter son eau :

L'ALÉNA ne crée aucun droit aux ressources en eau naturelle de l'une ou l'autre Partie. À moins d'être vendue dans le commerce et de devenir ainsi une marchandise ou un produit, l'eau sous toutes ses formes échappe entièrement aux dispositions de tout accord commercial, y compris l'ALÉNA. Or, rien dans l'ALÉNA n'oblige l'une ou l'autre Partie à exploiter son eau commercialement non plus qu'à commencer à l'exporter sous quelque forme que ce soit. L'eau qui se trouve à l'état naturel dans les lacs, les rivières, les réservoirs, les aquifères, les bassins hydrographiques, etc. n'est pas une marchandise ou un produit, ne se vend pas dans le commerce et, par conséquent, n'est pas et n'a jamais été visée par les conditions d'un accord commercial quel qu'il soit (Johansen).

Malheureusement, le contenu de ce communiqué n'a pas été entériné dans l'accord et il est possible qu'un amendement futur mette en cause d'importantes sommes d'argent liées au commerce et aux investissements bilatéraux. En 2001, Industrie Canada a soulevé la question dans un communiqué:

[...] étant donné les problèmes d'approvisionnement en eau des Etats-Unis, notamment dans le Sud-Ouest, il est presque assuré que la question des exportations d'eau surgira à nouveau. L'eau a été exclue de l'ALENA à condition qu'il n'y ait pas d'exportation en vrac. Mais la situation pourrait s'avérer intenable. Le Canada devrait revoir ses politiques en ce domaine et se demander comment il pourrait exporter de l'eau avec une politique de prix appropriée. Cette question soulèvera sans aucun doute un âpre débat, mais le temps est venu d'y accorder une certaine attention (Harris 39).

La signature et la mise à exécution des deux accords commerciaux (l'ALÉ et l'ALENA) reflètent non seulement une plus grande intégration commerciale avec les États-Unis, mais aussi un manque de détermination en matière d'eau. Étant donné que le Canada n'a pas formalisé sa position avec ses partenaires commerciaux, la question de l'eau demeure tout simplement en litige.

Le troisième moment, c'est le 23 mars 2005 avec la signature du PSP, connu aussi comme l'ALENA Plus. Cette entente est plus qu'un simple accord de libre-échange, puisque l'ALENA, d'après quelques analystes, avait déjà encadré le commerce avec les États-Unis (Pino, 2006). Par conséquent, à l'étape suivante, il s'agissait de faire front commun dans la région de l'Amérique du Nord par rapport à la sécurité et à l'approvisionnement en ressources naturelles. 


\section{Le PSP et l'eau}

La mise en cuvre du PSP en 2005 a servi à réaffirmer la volonté des partenaires de protéger les investissements et d'assurer l'exploitation des ressources naturelles telles que l'eau et le pétrole, entre autres. La déclaration mentionne que «Dans un monde qui évolue rapidement, on doit construire de nouveaux espaces de coopération dans le but d'offrir plus de sécurité à nos sociétés ouvertes, de rendre nos entreprises plus compétitives et nos économies plus solides » (Déclaration conjointe du Président Vicente Fox Quesada, du Président George W. Bush et du Premier ministre Paul Martin, le 23 mars 2005). Cet accord reconnait qu'il existe une étroite relation entre un approvisionnement en ressources naturelles assuré et la sécurité, ce qui mènera à la recommandation que l'on révise en profondeur certains domaines exclus de l'ALENA. Il est évident que cette révision inclura l'exportation de l'eau.

Le PSP permet aux signataires d'agir conjointement dans des secteurs stratégiques afin de contrer des menaces externes, d'accroître l'efficience et la sécurité de la circulation le long des frontières communes et d'assurer le contrôle des ressources naturelles telles que l'eau, le gaz et le pétrole. En effet, l'alliance porte sur six domaines: la mobilité du travail, la compétitivité, l'énergie, l'environnement (qui inclut l'eau, bien que les gouvernements affirment que l'eau ne fait pas partie des négociations), la sécurité et l'infrastructure frontalière et logistique. Les rencontres, tenues à huis clos, permettent à des représentants d'agences gouvernementales, à des intellectuels et à d'éminents hommes d'affaires des trois pays de discuter de questions de fond à l'extérieur des structures formelles, ce qui équivaut à un nouvel accord international en marge du pouvoir législatif des pays membres. Le but est de créer une nouvelle réglementation qui facilitera les investissements privés dans les espaces réservés à l'État. Les décisions prises au sein du PSP serviront à modifier la politique publique, ce qui risque d'avoir de sérieuses conséquences pour la souveraineté du Mexique et du Canada.

Les fondements de ce nouvel accord sont présentés dans le livre de Robert Pastor, universitaire américain, personne clé dans toutes les négociations, et promoteur d'une intégration économique de grande envergure à être réalisée par le biais d'institutions trilatérales oeuvrant dans les domaines de l'immigration, de l'énergie, du développement régional, de l'éducation, etc. L'idée d'une telle intégration a été renforcée par les attaques terroristes du 11 septembre 2001. Celles-ci ont fourni un argument de poids pour générer des accords visant une plus grande intégration pour assurer la sécurité des frontières des États-Unis. Le PSP a sans doute été très bien reçu par les hommes d'affaires, étant donné qu'il offre une plus grande sécurité pour leurs investissements. 
L'idée d'aller au-delà d'un simple accord de libre-échange a reçu l'approbation des trois gouvernements. Du côté canadien, Wendy Dobson du Howe Institute a qualifié de «big idea » la volonté du Canada et des États-Unis de poursuivre le développement d'accords stratégiques qui vont au-delà de l'intégration prévue par l'ALENA. Elle propose que l'on en fasse davantage pour harmoniser les politiques qui régissent la négociation de marchés communs de sorte à diluer l'indépendance politique des signataires de l'accord. Le Conseil canadien des chefs d'entreprises $(\mathrm{CCCE})^{11}$ qui représente les 150 plus grandes compagnies canadiennes a aussi donné un appui important à cette initiative. Ce conseil avait fait la promotion en 2003 d'une initiative relative à la sécurité et à la prospérité en Amérique du Nord et, une année plus tard, il a proposé un accord dont les fondements reposaient sur le principe de la sécurité de l'accès aux ressources ainsi que de leur approvisionnement.

Sur le plan des relations trilatérales, le Conseil des relations extérieures des États-Unis (Council on Foreign Relations), le CCCE et le Conseil mexicain des affaires internationales (COMEXI) ont présenté en 2005 un rapport conjoint, dans lequel on recommande le développement d'une stratégie commune pour l'ensemble de l'Amérique du Nord concernant la conservation des ressources naturelles, incluant évidemment les ressources énergétiques (le pétrole et le gaz) et l'eau (Construire une communauté nord-américaine). Cet accord n'a rien d'étrange, si on considère que deux des trois pays qui constituent cette région produisent d'importantes quantités de pétrole et qu'un des trois dispose d'importantes réserves d'eau.

Le Forum de l'Amérique du Nord, une structure parallèle au PSP, renforce le travail du PSP. Ce forum permet à des meneurs d'opinions, des dirigeants du monde des affaires, des universitaires et des dirigeants d'ONG, de rencontrer des représentants des gouvernements de trois pays de l'Amérique du Nord pour discuter de questions portant sur leur meilleure intégration. La deuxième rencontre du Forum de l'Amérique du Nord s'est déroulée à Banff au Canada du 12 au 14 septembre 2005. Parmi les participants figuraient le Secrétaire de la défense des États-Unis, Donald Rumsfeld, le Secrétaire mexicain de la Sûreté publique, Eduardo Medina Mora, et le Commandant des Forces armées canadiennes, le Général Rick Hillier. Des cadres d'entreprises telles Pétroleos Mexicanos, Chevron, Suncor Energy et Lockheed Martin étaient aussi de la partie (Gonzalez Amador).

Au sommet des chefs d'États de l'Amérique du Nord à Cancun en 2006, on a convenu d'encourager les chefs d'entreprises des trois pays de se donner une structure qui leur permettrait de discuter de la complémentarité de l'accroissement de la coopération économique et de la sécurité dans les prises de décision en matière de politique et de réglementation. Le résultat a été la mise sur pied du Conseil nord-américain de la compétitivité (CNAC) 
composé de 10 dirigeants d'entreprises de chacun des trois pays chargés de représenter leurs secteurs privés respectifs. Lors de la première rencontre du CNAC à Montebello au Québec le 21 août 2007, on a adopté le rapport intitulé «Construire une Amérique du Nord sécuritaire et compétitive» énumérant 51 recommandations portant sur des objectifs à être réalisés en 2007,2008 et 2010 dans les domaines de la circulation transfrontalière, les normes et la réglementation, et l'intégration de l'énergie (NACC 1-2).

Il n'est pas surprenant que le pétrole et le gaz figurent à l'ordre du jour des rencontres de ces organismes, puisque leur production était à la baisse aux États-Unis, tandis qu'au Canada (en particulier en Alberta) et au Mexique, il existe un grand potentiel de développement dans ces secteurs. On estime que le Canada est le deuxième pays au niveau mondial en termes de réserves prouvées, de sorte que l'on prévoit la possibilité de tripler sa production et ses exportations d'ici 2025. Il ne serait pas non plus surprenant qu'à l'avenir, l'approvisionnement en eau soit ajouté à l'ordre du jour de ces mêmes forums de discussion. Puisque certaines régions des États-Unis, comme le Texas et la Californie souffrent déjà de stress hydrique il est tout à fait prévisible que les États-Unis se tournent vers leurs voisins, comme ils l'ont fait dans le cas du pétrole.

Le projet le plus récent qui a réuni des décideurs des trois pays de l'Amérique du Nord a été conçu par le Center for Strategic and International Studies (CSIS) des Etats-Unis. Le North American Future 2025 Project a reçu la collaboration du Conference Board of Canada et du Centro de Investigacion y Docencia Economicas (CIDE) du Mexique. Selon ses auteurs, ce projet permettra aux décideurs des trois pays concernés d'adopter des politiques éclairées, stratégiques et de longue portée :

[...] with an emphasis on regional integration. Specifically, the project will focus on a detailed examination of future scenarios, which are based on current trends, and involve six areas of critical importance to the trilateral relationship: labor mobility, energy, the environment, security, competitiveness and border infrastructure and logistics. (CSIS 2)

La question du partage de l'eau douce occupe une place d'honneur dans le devis de projet. Compte tenu des changements prévus en ce qui a trait à la disponibilité, à la qualité et à l'allocation de l'eau douce avant 2025, les décideurs devront faire preuve de créativité dans leur façon d'aborder les questions portant sur la gestion transfrontalière de l'eau douce. Selon les auteurs :

One such option could be regional agreements between Canada, the United States, and Mexico on issues such as water consumption, water transfers, artificial diversions of fresh water, 
water conservation technologies for agricultural irrigation, and urban consumption. (CSIS 6)

Les dirigeants des trois pays devront faire en sorte que leurs systèmes politiques et légaux respectifs n'entravent pas l'atteinte du but primordial de l'avenir pour l'Amérique du Nord, à savoir : « to achieve joint optimum utilization of the available water and to implement procedures that will help avoid or resolve differences over water in the face of ever-increasing pressures over this priceless resource. » (CSIS 6-7). La question de l'eau déborde le cadre économique. Comme l'a précisé le rapport intitulé Global Water Future préparé par le (CSIS) et Sandia National Laboratories en 2005, l'eau doit figurer dans la politique humanitaire du gouvernement américain qui comprend les intérêts de ce dernier en matière de sécurité politique, sociale, économique et commerciale. Les diverses déclarations du gouvernement américain sont claires en ce qui concerne l'eau et la nécessité de l'inclure parmi ses intérêts stratégiques, ce qui explique pourquoi l'eau canadienne est devenue une priorité bien définie dans les négociations du PSP.

\section{L'eau canadienne est-elle protégée?}

Puisque l'eau canadienne est une ressource stratégique pour les États-Unis, il y a lieu de savoir si celle-ci est bien protégée par les lois canadiennes. Selon l'OCDE, le Canada, un pays riche en eau, utilise seulement 1,6\% du total de cette ressource renouvelable, et ce, en dépit du fait que la consommation par habitant est de l'ordre de $1,420 \mathrm{~m}^{3} /$ année, un des taux de consommation les plus élevés au monde. La consommation par habitant aux États-Unis est de l'ordre de $1,790 \mathrm{~m}^{3} /$ année tandis qu'en France, elle se . situe à $530 \mathrm{~m}^{3} /$ année et au Royaume-Uni à $340 \mathrm{~m}^{3} /$ année $^{13} 14$.

De prime abord, la question de l'eau ne devrait pas poser de problèmes pour les États-Unis puisque leur richesse en eau renouvelable est à peu près équivalente à celle du Canada. Cependant, il y a un problème sérieux puisque ses 290 millions d'habitants ont le taux de consommation d'eau le plus élevé au monde et les besoins en eau de ses industries augmentent constamment. Bien que le gouvernement américain ait mis en œuvre des projets visant la conservation et le recyclage de l'eau, comme c'est le cas au Texas où on a réduit la consommation depuis 1980, ou en Californie qui maintient sa consommation en eau aux niveaux de 1970 en dépit du fait que sa population ait doublé, les besoins en eau sont imminents et importants. Force est de constater que l'eau du Canada prend une valeur pour le gouvernement américain, spécialement dans le cadre du PSP.

Quand on parle des grandes réserves d'eau du Canada, il ne faut pas oublier que l'accès à cette eau est difficile puisque la plupart des rivières au Canada coulent vers le nord, dans une zone semi-aride et peu peuplée. À ces conditions géographiques et climatiques peu favorables s'ajoute le fait que 
la plus grande partie de la population canadienne habite le long de la frontière avec les États-Unis, où la demande d'eau est la plus importante et où l'eau est la plus contaminée. Toutefois, notre réflexion doit nous mener au-delà de la simple disponibilité de l'eau; il faut se pencher sur la position du gouvernement canadien par rapport aux exportations d'eau depuis les quarante dernières années et sur les défis auxquels il devra faire face pour composer avec les besoins de son voisin du sud, lequel sera aux prises avec une sérieuse pénurie d'eau à court terme.

Même si depuis les années soixante on a toujours rejeté l'idée de méga projets comme la construction d'un aqueduc pour transporter de l'eau aux États-Unis, la possibilité d'exporter de l'eau a toujours été présente. Deux genres d'arguments sont toujours invoqués: Premièrement, du point de vue strictement économique, ces méga projets ne profiteraient qu'à quelques régions (comme ça été le cas récemment avec le pétrole en Alberta). Deuxièmement, on a soulevé les aspects humanitaires. Quoique le gouvernement fédéral se soit toujours opposé à l'exportation de l'eau; sa position n'a jamais été très claire et convaincante. Cette position contenue dans des affirmations ambiguës et les réponses évasives de différents ministères, n'a jamais précisé si la question de l'eau relevait ou non de la sécurité nationale. Cette ambiguïté a probablement toujours eu pour objet de ne pas entraver la relation commerciale du Canada avec les États-Unis.

Un autre élément contribue à la nébulosité de la position canadienne: le manque d'informations précises sur les eaux souterraines ${ }^{15}$, ce qui contribue au mythe selon lequel le Canada disposerait de beaucoup d'eau. En 2005, un groupe d'experts a fait une série de recommandations au gouvernement du Canada parmi lesquelles figurait la nécessité de dresser une carte topographique de tous les aquifères du territoire canadien avant 2010. Parmi les autres recommandations, on retrouve: évaluer la disponibilité et les quantités d'eau; mettre sur pied (par le gouvernement fédéral) une banque de données centralisée de l'eau; s'assurer que la question du renouvellement de l'eau figure dans les études; soutenir financièrement l'Institut national de recherche sur les eaux; mettre sur pied un Conseil national de l'eau (qui inclurait des représentants de l'industrie, des centres de recherche et d'autres instances du gouvernement liées à l'eau), dont la tâche serait l'identification de problèmes relevant du gouvernement fédéral; et finalement, formuler des stratégies destinées à résoudre les problèmes liés à l'eau (Water in the West: Under Pressure). Ces recommandations s'avèrent fondamentales non seulement pour connaître avec exactitude la disponibilité des ressources hydriques en territoire canadien, mais aussi pour unifier les critères régissant la prise de décisions et l'élaboration de politiques fédérales et d'accords interprovinciaux.

Présentement, il n'y a pas de loi fédérale pour réglementer la gestion de l'eau douce, une ressource provinciale, sur le territoire canadien. La 
répartition constitutionnelle des pouvoirs entre les niveaux fédéral et provincial a anticipé les défis liés à la navigation, à la pêche et, récemment, à la production d'électricité, mais pas ceux liés à l'exportation de l'eau, au maniement des bassins et des rivières, etc. Sans un cadre législatif, il sera difficile pour les gouvernements fédéral et provinciaux de conclure des ententes pour faire face aux défis à l'horizon. Même si le gouvernement fédéral a la responsabilité des relations internationales et doit légiférer lorsqu'il s'agit de l'intérêt national, puisque la gestion des ressources naturelles relève du domaine provincial, il sera toujours nécessaire de tenir compte des attentes et des besoins des provinces lorsqu'il s'agira de réglementer la gestion et les exportations d'eau. Il sera important de persuader les provinces de signer des accords régissant la protection de l'eau afin d'éviter les actions unilatérales.

L'adoption du projet de loi fédéral C-156 de 1988, dont il a été question plus haut, aurait, tout en interdisant les exportations massives d'eau, facilité la signature d'ententes pour l'exportation par les provinces de petites quantités d'eau. C-156 aurait régi autant les projets entrepris par le secteur privé que par le secteur public. Présentement, certaines provinces ont mis sur pied des projets d'exportation d'eau, mais sans beaucoup de succès.

En l'absence d'une loi fédérale, certaines provinces légifèrent en matière de gestion de l'eau. Le Québec, par exemple, a mis en place quelques initiatives par rapport à la protection de l'eau sur son territoire ${ }^{16}$. En 1996, Terre-Neuve-et-Labrador a adopté une politique qui permettait l'exportation d'eau en vrac par bateau en tenant compte, bien entendu, des questions environnementales et des bénéfices économiques pour la province. De son côté, l'Ontario a considéré la possibilité d'exporter de l'eau en 1998.

Entre-temps, Environnement Canada poursuit une politique de protection écologique des bassins, des rivières, etc., laquelle exige des accords politiques avec les provinces. En 1999, le gouvernement fédéral a proposé une entente de coopération orientée vers l'interdiction de l'exportation d'eau, selon laquelle les provinces devaient modifier certaines de leurs lois. Cet accord, signé sans beaucoup d'enthousiasme par les provinces, n'a pas eu l'impact souhaité. Le manque de régulation et de coopération en matière d'eau entre le fédéral et le provincial se conjugue avec un autre élément, celui de gérer le double discours en ce qui concerne l'eau. D'un côté, le gouvernement fédéral a persuadé sa population que l'eau canadienne est une ressource importante, qu'il faut bien la gérer et qu'elle appartient à tous les citoyens. De l'autre côté, il n'y a pas de déclaration formelle portant sur la souveraineté canadienne en matière de l'eau, et ce, en dépit du fait que celle-ci aitété exclue de l'ALENA et du PSP. Il est possible que cette ambiguïté soit utilisée comme moyen de permettre l'accès sans limitations au marché américain et pour éviter des 
confrontations avec ce voisin du sud. Toutefois, le manque de clarté dans les engagements internationaux, tels l'ALENA et le PSP, fait en sorte que le sort de l'eau du Canada sera décidé dans d'autres forums dans lesquels les États-Uniens auront gain de cause.

Pour le moment, la politique du gouvernement en matière de gestion et d'exportation d'eau douce est celle énoncée par le ministre canadien de 1'Environnement dans un communiqué de presse en date du 13 avril 2007 où il déclare:

Le gouvernement du Canada n'a aucune intention d'entreprendre des négociations, que ce soit à huis clos ou autrement, relativement à l'exportation de grandes quantités d'eau. Des restrictions sont en vigueur au Canada pour interdire les prélèvements massifs d'eau, y compris par détournement, lesquelles sont doublées d'amendes et de peines d'emprisonnement sévères. Le Canada est déterminéà protéger l'eau dans son état naturel et à préserver l'intégrité des écosystèmes, et continuera de le faire. (Environnement Canada)

Le ministre Baird poursuit :

Au Canada, la responsabilité de la gestion de l'eau repose sur plusieurs ordres de gouvernement. Les provinces et les territoires du Canada sont la principale compétence quant à la plupart des aspects de la gestion et de la prōtection de l'eau, et ils ont adopté des lois, des règlements ou des politiques interdisant le prélèvement d'eau à grande échelle aux fins d'exportation hors des eaux provinciales et territoriales. (Environnement Canada)

En dépit de la déclaration du ministre Baird, il y a toujours pas mal d'incertitude et, devant une telle incertitude, le gouvernement fédéral devrait poursuivre, avec tous les intervenants, un dialogue sur une politique de protection de l'eau sur tout son territoire. Il convient d'indiquer que jusqu'à présent le manque de précision en matière d'eau a donné de bons résultats en autant que nos bonnes relations avec les États-Unis n'ont pas souffert.

\section{Conclusion}

L'histoire de la gestion de l'eau canadienne et les divers accords conclus avec les États-Unis démontrent que jusqu'à présent, c'est le gouvernement américain qui a dicté les enjeux et orienté le dossier. Effectivement, au fil des années, les États-Unis ont amené le Canada à considérer l'eau d'abord comme un bien économique - avec l'exportation de l'eau embouteillée - et ensuite comme une composante de sa politique extérieure, particulièrement dans les négociations dans le cadre du PSP. Toutefois, le Canada a toujours beaucoup de difficultés à établir des limites précises pour la gestion de l'eau à l'interne et à l'externe. Il est toujours aux prises avec la complexe réalité du partage des responsabilités fédérale-provinciales dans le domaine et 
avec sa préoccupation de longue date de maintenir de bonnes relations politiques et commerciales avec les États-Unis.

Ce sont les besoins hydriques des États-Unis qui semblent avoir priorité dans les discussions portant sur le partage et la gestion de l'eau en Amérique du Nord et malheureusement, les forums où se déroulent ces discussions ne se prêtent pas à la transparence et à l'ouverture, ce qui risque d'aboutir à des prises de décisions en marge des processus démocratiques et constitutionnels canadiens. Compte tenu des conséquences à long terme des décisions et du contexte constitutionnel canadien, il faudra chercher à harmoniser le contexte décisionnel avec le partage des responsabilités entre les gouvernements fédéral et provinciaux et faire preuve de plus de transparence afin que les citoyens soient en mesure de connaître les enjeux et faire des choix éclairés.

De plus, si la disponibilité de l'eau est véritablement une question de sécurité, les trois pays devront ajouter à leurs discussions les questions telles l'amélioration de la qualité de l'eau, sa conservation et les besoins en infrastructure. Présentement, il n'y a pas d'initiatives orientées vers une politique commune de conservation ni de culture commune de consommation.

Le Canada, grâce à sa longue expérience en matière de conservation de l'eau, pourrait proposer aux États-Unis un agenda plus vaste pour une meilleure conservation. Résoudre le problème de l'eau, c'est résoudre un problème à la fois environnemental et commercial, et cela implique non seulement de longues discussions et l'adoption d'une éthique différente mais aussi une conception différente de l'intégration continentale. Enfin, pour réussir dans ce domaine, le gouvernement canadien devra, en collaboration avec les gouvernements provinciaux, élaborer une politique hydrique intégrée et claire.

\section{Notes}

1. La valeur du commerce des biens et des services s'élève à environ 1,800 millions de dollars annuellement, tandis que l'activité transfrontalière est d'un demi million de personnes et de 37,000 camions par jour. 79 p. 100 des exportations et 65 p. 100 des importations canadiennes se font avec les États-Unis. (DFAIT, 2005 19)

2. Compte tenu de l'importance du commerce entre les deux pays et afin d'assurer la sécurité frontalière, on a conçu la frontière intelligente dont l'objectif est de permettre aux agents de l'immigration américaine de certifier les marchandises canadiennes destinées à l'exportation sur place dans les usines canadiennes. (Montero, D., 2002)

3. L'élection de M. Harper et de son Parti conservateur à la fin de 2005 a mis fin à 12 années de gouvernement libéral. Le Parti libéral est un parti du centre qui a mené les destinés canadiennes durant 78 des 110 années de l'histoire électorale canadienne. On a appelé le Parti conservateur le parti républicain canadien en 
vertu des similitudes avec les républicains américains : conservatisme social, fondamentalisme religieux et une base rurale. Le gouvernement de M. Harper, a remporté les élections en s'attaquant à l'inefficacité et à la corruption des Libéraux. Sa devise, « un gouvernement responsable réussira à faire des choses pour tous ", lui a permis de mettre en cuvre son programme de droite.

4. Récemment, le Premier ministre Harper a décidé que le Protocole de Kyoto était une erreur, bien que les groupes environnementalistes estiment que cette nouvelle position canadienne soit injustifiable. http://www.ledevoir.com/2007/11/27/ $166236 . \mathrm{html} ? \mathrm{fe}=2526 \& \mathrm{fp}=179622 \& \mathrm{fr}=54540$.

5. L'agriculture, les forêts et la pêche représentent 13,6 p. 100 du PIB canadien. De plus, les exportations de bois du Canada représentent 40 p. 100 du total mondial et ses exportations de blé, de poissons, d'énergie et de minéraux sont considérables. (OCDE, 2004 15)

6. Les Grands Lacs constituent $18 \%$ de l'eau douce du monde. 40 p. 100 de la frontière de $8000 \mathrm{~km}$ entre les États-Unis et le Canada est démarquée par les Grands Lacs.

7. Une des plus grandes controverses que l'on retrouve dans ces traités est le désaccord entre le Canada et les États-Unis par rapport aux déviations de l'eau du Lac Michigan vers le fleuve Mississippi.

8. Il faut signaler que toute déviation de l'eau des Grands Lacs pourrait avoir d'importantes répercussions pour le Canada, puisque les rivières qui sont liées à cette grande réserve d'eau coulent vers le Nord du Canada. Ces déviations pourraient modifier le débit d'eau des rivières ainsi que les habitats de nombreuses espèces.

9. Les États-Unis, dont la population représente 5 p. 100 de la population mondiale, consomment 25 p. 100 des ressources naturelles du monde. Environ 40 p. 100 de l'industrie américaine et 50 p. 100 de l'industrie canadienne se trouvent dans la région des Grands Lacs. À ces données, il faudrait ajouter que quelques régions des États-Unis telles que la Californie et l'Arizona souffrent présentement d'une insuffisance hydrique.

10. Il faut signaler qu'au Canada, les ressources naturelles font les frais d'un grand débat depuis longtemps, mais des sujets tels que la pollution de l'air ou de l'eau ont surgi comme des thèmes de politique publique jusqu'aux années soixante. (Dwivedi et al.)

11. Le CCCE, créé en 1976, est reconnu comme l'organisme commercial le plus influent du Canada. Il a la responsabilité d'appuyer les politiques publiques pour renforcer l'économie canadienne en Amérique du Nord et dans le reste du monde. Ses membres administrent plus de 2,3 billions de dollars canadiens en actifs lesquels rapportent des profits annuels de l'ordre de 600 milliards de dollars canadiens, ce qui représente une partie très importante des investissements, des exportations, de la recherche et du développement du secteur privé. http://www.ceocouncil.ca/fr/view/?document_id=366\&area_id=7.

12. En 1991, les États-Unis produisaient 9,1 millions de barils de pétrole par jours alors qu'en 2005 sa production passait à 7,61 millions de barils par jour, reléguant les États-Unis au $4^{\mathrm{e}}$ rang mondial pour la production de cette ressource. Voir Bulletin Informatif du CEDEFNA, mai 2006, http://csh.izt.uam.mx/cen_doc/ cedefna/.

13. L'OCDE considère aussi que le Canada gère mal son eau. Cette mauvaise gestion serait attribuable au bas prix de l'eau par rapport aux pays développés. OCDE, 2004. 
14. Le Mexique, le troisième partenaire de l'ALENA et du PSP ne dispose que de 1 p. 100 du total mondial d'eau. Sa population est de 100 millions d'habitants et sa consommation par personne est de $731 \mathrm{~m}^{3} /$ année.

15. Dans une entrevue avec des membres du personnel d'Environnement Canada, on a signalé qu'on ne savait pas avec exactitude combien d'eau souterraine il y avait au Canada.

16. Cette province a fait connaître en 2002 sa Politique nationale de l'eau, qui se base sur une consultation publique menée par le Bureau d'audiences publiques sur l'environnement. L'orientation des politiques en ce qui concerne l'eau interdit le transfert massif d'eau de surface ou souterraine à l'extérieur du Québec, sauf certaines exceptions pour des questions humanitaires ou d'intérêt public. De même, tout engagement international en rapport avec l'eau doit se soumettre à l'approbation de l'Assemblée nationale du Québec.

\section{Bibliographie}

Canadian Council of Chief Executives (CCCE). http://www.ceocouncil.ca/fr/view/ ?document_id=366\&area_id $=7$.

Canadian Council of Chief Exxecutives (CCCE). Nouvelles frontières : Bâtir un partenariat Canada-États-Unis pour le $21^{e}$ siècle en Amérique du Nord, avril 2004, http://www.ceocouncil.ca/fr/view/?document_id=366\&area_id=7.

CEDEFNA, Boletin Informativo, http://csh.izt.uam.mx/cen_doc/cedefna, México, mayo 2006.

Center for Strategic and International Studies, www.sandia.gov/water/docs/ CSIS-SNL OGWF 9-28-05.PDF.

Center for Strategic and International Studies (CSIS), North American Future 2025 Project, http://www.canadians.org/water/documents/NA_Future_2025.pdf.

Center for Strategic and International Studies (CSIS) and Sandia National Laboratories, Global Water Futures: Addressing Our Global Water Future, September 30, 2005, http://www.sandia.gov/water/docs/CSIS-SNL_OGWF_928-05.PDF.

Clarkson, Stephen, en Gutiérrez H. Teresa, Verea, M (Coord), Canadá en Transición, CISAN, México, 1994.

Construire une communauté nord-américaine: Rapport du Groupe de travail indépendant sur l'avenir de l'Amérique du Nord, 2005, http://www.rqic. alternatives.ca/psp/northamerica tf_final_fr.pdf.

Déclaration conjointe du Président Vicente Fox Quesada, du Président George W. Bush et du Premier Ministre Paul Martin, le 23 mars 2005, http://www.sre. gob.mx/eventos/aspan/faqs.htm.

DFAIT, Declaración de política internacional de Canadá. Nuestro rol internacional: un sentimiento de orgullo $e$ influencia en el mundo, ww.dfait-maeci.gc.cal cip-pic/, 2005.

Dobson, Wendy, "Shaping the Future of the North American Economic Space: A Framework for Action", Toronto: C.D., Howe Institute Commentary, No. 162, The Border Papers, April 2002, de Gary C. Hufbauer y Jeffrey J. Schott, The Prospects for Deeper North American Economic Integration: a US Perspective, C.D: Howe Institute, No. 195, January $2004 \mathrm{http} / /$ www.petersoninstitute.org/ publications/papers/hufbauer0104.pdf.

Dwivedi, O.P., et al, Sustainable Development and Canada. National and International Perspective, Broadview Press, Canada, 2001.

Environnement Canada, Communiqué, 13 avril 2007 : Le Canada n'a aucune intention de négocier des exportations de grandes quantités d'eau, http://www.ec.gc.ca/ default.asp?lang=Fr\&n=714D9AAE-1 \&news=B362E955-305B-49FB-BA8BAD6292238387.

González Amador, Roberto, Reunión secreta para " profundizar la integración 》 de América del Norte, La Jornada, 25 de septiembre 2006, http://www.jornada. unam.mx/2006/09/25/003n1 pol.php. 
Gouvernement du Québec, Politique nationale de l'eau. L'eau. La vie et l'avenir. Bibliothèque nationale du Québec, Québec 2002.

Harris, Richard G., L'intégration économique de l'Amérique du Nord : problématique et recherche future, $\mathrm{N}^{\mathrm{c}} 10$, Université Simon Fraser, avril 2001, http://strategis.ic.gc.ca/epic/site/eas-aes.nsf/fr/ra01809f.html.

Hufbauer, Gary C. et Jeffrey J. Schott, The Prospects for Deeper North American Economic Integration: a US Perspective, C.D: Howe Institute, No. 195, January 2004, http://www.petersoninstitute.org/publications/papers/hufbauer0104.pdf.

Johansen, David, Les exportations d'eau et l'ALENA, Division du droit et du gouvernement, Gouvernement du Canada, 8 mars 1999, http://dsp-psd.tpsgc. gc.ca/Collection-R/LoPBdP/EB/prb995-f.htm.

Lasserre, F., et Descroix, L., Eaux et territoires. Tension, coopérations et géopolitique de l'eau. Presses de l'Université du Québec, Canada, 2005.

Le Devoir, http://www.ledevoir.com/2007/11/27/166236.html?fe=2526\&fp=179 $622 \& \mathrm{fr}=54540$.

Montero, D., 2002, " Interprovincial Trade and Intelligent Borders in Canada », dans Voices of Mexico, $\mathrm{n}^{\circ} 61$ octobre-décembre, México, $\mathrm{p}$ 64-68.

2003, "Los vínculos de Canadá y México a partir de sus inversiones directas ", en Vidal y Guillén (Coord), La economía mexicana bajo la crisis de Estados Unidos, Ed. UAM-Miguel Angel Porrúa, México.

, "Water in Québec: A Transnational Business », dans International Journal of Canadian Studies, $\mathrm{n}^{\circ}$ 29, 2004.

, «Les Grands Lacs et le Saint-Laurent face aux défis du développement et des nouveaux besoins en eau », communication présentée à Someure, France, juin, 2006.

, "Agua y transnacionales: una historia empresarial exitosa ", 2007, sous presse.

Niosi, Jorge, Les multinationales canadiennes, Ed. Boréal Express,1982, Montréal.

North American Competitiveness Council (NACC), Communiqué : Le conseil commercial trilatéral réclame des progrès dans le renforcement de la sécurité et de la compétitivité de l'Amérique du Nord, http://www.ceocouncil.ca/publications/ pdf/test_760c27d08e7dbf866c801b81415d10a9/Communique_CNAC_21_aout 2007.p̄ff.

Orgañisation de coopération et de développement économiques (OCDE), Environmental Performance Reviews, France, 2004.

Pastor Robert, Toward a North American Community, Institute of International Economics, Washington, D.C., 2001.

Pino, Enrique, « Canadá y México: ¿socios distantes? Los limites del comercio intraregional ", communication présentée au Seminario Interuniversitario de Estudios Canadienses (SEMINECAL), Bogotá, les 6 et 7 avril 2006.

Secretaría de Relaciones Exteriores, « Alianza para la Seguridad y Prosperidad de América del Norte », http://www.sre.gob.mx/eventos/aspan/faqs.htm.

Serfati, Claude, Conférence à la UAM, «Impérialisme et militarisme: actualité du XXI siècle $", 3$ mai 2006.

Water in the West: Under Pressure. Fourth Interim Report of Standing Senate Committee on Energy, the Environment and Natural Resources, November 2005, http://www.parl.gc.ca/38/1/parlbus/commbus/senate/com-e/enrg-e/rep-e/rep13n ov05-e.htm\#Water_Under_Pressure. 